\title{
Uso del espacio por adultos de gaviota patia- marilla Larus michahellis Naumann, 1840 durante el periodo reproductor: resultados preliminares en relación al uso de vertederos.
}

\section{Spatial use by adult yellow-legged gulls Larus michahellis Naumann, 1840 during the breeding period: preliminary results in relation to the use of landfills.}

Juan Arizaga ${ }^{*}$, Maite Laso $^{1}$, Nere Zorrozua', Sergio Delgado ${ }^{1}$, Asier Aldalur', Alfredo Herrero ${ }^{1}$

\section{Resumen}

En la actualidad, con el progresivo cierre de vertederos, nos situamos en un marco experimental ideal para estudiar cómo afecta la reducción drástica de un recurso trófico en la ecología, dinámica poblacional y movimientos de una especie con alto interés en la gestión, como es la gaviota patiamarilla Larus michahellis Naumann, 1840. En este contexto, en 2016 se equiparon con GPS un total de 10 ejemplares adultos de gaviota patiamarilla en una de las colonias de cría de Gipuzkoa, de los que sólo 4 proporcionaron datos hasta el 31.07.2016. El análisis preliminar aporta información básica sobre el uso del territorio durante una parte del periodo reproductor. El porcentaje de localizaciones se distribuyó de esta manera: 60\% en la colonia; 18\% en otro tipo de hábitats; $12 \%$ en prados y cultivos; y $6.5 \%$ en vertederos. Se detecta, así, un relativo alto uso de los prados y cultivos del entorno de la colonia, así como de dos de los vertederos más importantes de la región. Por otro lado, parece existir alta variabilidad individual en el uso del territorio que podría reflejar diferentes estrategias en la búsqueda de alimento.

Palabras clave: Dispositivos GPS, ecología espacial, especies oportunistas, Gipuzkoa, vertederos.

\footnotetext{
${ }^{1}$ Departamento de Ornitología, Sociedad de Ciencias Aranzadi.

Zorroagagaina 11, 20014 Donostia. 


\begin{abstract}
Today, the progressive closure of landfills provides a good opportunity to study how this affects the drastic reduction of a food resource on the ecology, population dynamics and movement patterns of opportunistic species, such as the yellow-legged gull Larus michahellis Naumann, 1840. Against this background, in 2016, we captured 10 adult breeding gulls in one of the main breeding colonies in Gipuzkoa. These individuals were equipped with a GPS device. Only four of them provided data up to the end of the breeding period (31.07.2016). Preliminary analyses provide basic information about spatial use and trophic habitat during the breeding period (colony: 60\%; other habitat types: 18\%; grasslands/crops: 12\%; dumps: 6,5\%). An apparently relatively high use of grasslands and crops is confirmed, as well as the use of two of the largest landfills in the region. We also observed a very high individual variation in territory use, which might reflect different foraging strategies.
\end{abstract}

Key words: GPS device, spatial ecology, opportunistic species, Gipuzkoa, dumps.

\title{
Laburpena
}

Egun, urratsez urrats zabortegiak ixten ari direlarik, testuinguru esperimental ezin hobean gaude ikertzeko nola eragiten dion baliabide trofiko baten erabateko murrizketak maila ekologikoan, populazio dinamikan eta mugimenduetan, baliabide horren kudeaketarekin lotura handia duen kaio hankahoriari, Larus michahellis Naumann, 1840.

Testuinguru honetan, 2016. urtean Gipuzkoako ugaltze-kolonia bateko 10 ale helduri GPS gailuak jarri zitzaizkien. Horietatik lauk soilik eman zituzten datuak 2016.07.31 ra arte. Atariko analisiek ugaltze-garaian lurraldea nola erabiltzen duten oinarrizko informazioa ematen dute. Kokapen portzentajeak honela banatu ziren: \%60 kolonian, \%18 beste mota bateko habitatetan; $\% 12$ belardi eta labore eremuetan; eta \%6.5 zabortegietan. Eta, horrela, koloniaren inguruko belardi eta labore eremuen zein herrialdeko bi zabortegi garrantzitsuren erabilera altua behatu da. Bestalde, banaka, lurralde erabileran aldakortasun handia dagoela dirudi, eta horrek elikagaiak bilatzeko estrategia desberdinen erabilera iradoki dezake.

Gako hitzak: GPS gailuak, espazio-ekologia, espezie oportunistak, Gipuzkoa, zabortegiak.

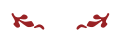

\section{Introducción}

Muchas especies animales oportunistas son capaces de explotar recursos tróficos de origen humano, tales como la basura que se deposita en los vertederos o los descartes que resultan de la actividad pesquera (Bertellotti et al., 2001; Granadeiro et al., 2014). La explotación de este tipo de recursos (abundantes y predecibles en el tiempo) ha propiciado a menudo cambios notables en el comportamiento y dinámica de las poblaciones que los utilizan 
(Steigerwald et al., 2015; Castège et al., 2016; Gilbert et al., 2016). Por ejemplo, varias especies de gaviotas en todo el mundo han incrementado el tamaño de sus poblaciones muy significativamente (Olsen \& Larson, 2004). Tal incremento ha generado, en ocasiones, la aparición de problemas ecológicos, sanitarios, sociales o económicos (Raven \& Coulson, 1997; Barras \& Wright, 2002; Oro et al., 2005; Oro et al., 2013). La gestión del binomio vertederosgaviotas se ha convertido, en consecuencia, en algo prioritario para las administraciones y organismos gestores de residuos sólidos urbanos (Smith \& Carlile, 1993; Harris \& Wanless, 1997).

En el Paleártico sudoccidental una de las especies más comunes de gaviota es la patiamarilla L. michahellis Naumann, 1840, cuya distribución abarca las costas de España y Portugal, el Mediterráneo occidental, el noroeste de África y la Macaronesia (Olsen \& Larson, 2004). La especie también aparece en algunas zonas húmedas de Centroeuropa y la costa oeste de Francia (Geroudet, 1984; Yésou, 1991). Igualmente, también se observa como reproductora en el interior de países de la región mediterránea, como España (Molina, 2009). En toda su área de distribución, su población se estima en unas 150.000200.000 parejas (Olsen \& Larson, 2004). En el Cantábrico oriental (País Vasco) se ha calculado una población de unas 4.200 parejas, que durante las décadas de 1980 a 2000 se incrementó en casi un 150\% (Arizaga et al., 2009), si bien en la última década se ha registrado un descenso notable en algunas colonias, aparentemente en paralelo al cierre de vertederos (Galarza, 2015).

En la actualidad, con el progresivo cierre de vertederos, nos situamos en un marco experimental ideal para estudiar cómo afecta la reducción drástica de un recurso trófico en la ecología, dinámica poblacional y movimientos de una especie con alto interés en la gestión, como es la gaviota patiamarilla (Oro et al., 1995; Duhem et al., 2002). En este artículo describimos la distribución espacial durante el periodo de cría. Con carácter preliminar, mostramos datos obtenidos tras el comienzo de un proyecto de marcaje de adultos reproductores mediante dispositivos GPS en una de las colonias de cría en Gipuzkoa.

\section{Material y métodos}

\section{Área de estudio y marcaje de ejemplares}

El estudio se realizó con ejemplares capturados y marcados en una de las colonias de cría de la especie en Gipuzkoa (Ulía, Donostia, 43020'N 01057'W). Se estima que la colonia está formada por unas 500 parejas (Arizaga et al., 2009).

Durante el periodo de cría de 2016, entre los meses de mayo y junio (24.05.2016-02.06.2016) se capturaron mediante trampas cepo un total de 10 ejemplares adultos durante la incubación, esto es, cuando todos ellos tenían huevos. Tras su captura los ejemplares se anillaron con una anilla metálica (remite Aranzadi) y con una anilla de PVC para su lectura a distancia. Además, se midieron las siguientes variables biométricas (para más detalles ver 
Arizaga et al., 2008): longitud de cráneo+pico, cúlmen, alto de pico, distancia de la punta del pico hasta el gonys y tarso. El sexo se estableció mediante la aplicación de la ecuación dada en Arizaga et al. (2008) que considera las medidas morfológicas anteriores. Finalmente, a cada ejemplar se le colocó un GPS en la zona dorsal (manto), mediante un arnés de teflón, ajustado a la medida de cada ejemplar. El peso del GPS con el arnés nunca superó el 5\% del peso del ave.

El GPS se programó para determinar la posición del ave cada 30 minutos durante $24 \mathrm{~h}$. Los dispositivos en cuestión son prototipos producidos por Wimbitek, S. L. Disponen de un GPS (que nos proporciona longitud, latitud y altitud; modelo Gtop fgpmmopa6h), microprocesador, memoria, paneles solares y batería, pero todavía carecen de acelerómetro, por lo que sólo se obtienen datos sobre la posición geográfica de cada ejemplar, pero se desconoce si éste estaba posado o en vuelo (Bouten et al., 2013). Además, cada dispositivo cuenta con un emisor por radio que se comunica periódicamente con una antena colocada en la colonia que permite el volcado de datos y, potencialmente, la actualización de los parámetros que determinan la frecuencia de toma de posiciones.

\section{Hábitats}

En el caso de Gipuzkoa, la especie se alimenta tanto de residuos depositados en vertederos como de presas de origen marino e invertebrados capturados en pastizales y cultivos (Arizaga et al., 2013b). Para determinar el uso del territorio se definieron, en concordancia con los hábitat más seleccionados por la especie, los siguientes: marino (posiciones en -sobre- el mar), vertedero (posiciones en vertederos, que fueron los de Sasieta en Beasain y Zaluaga en Senpere; Fig. 1; en ambos casos se consideró un radio de 500 m en torno al centroide del vertedero), prado (posiciones en prados y cultivos), colonia (se incluyeron aquí posiciones tanto en la misma colonia como a su alrededor -buffer de $500 \mathrm{~m}$ en torno al centro de la colonia), otros (posiciones en el resto de hábitats, no incluidos en las categorías anteriores). En el caso de los vertederos, el buffer de $500 \mathrm{~m}$ abarca una superficie algo superior a la de éstos con el fin de incluir también las zonas del entorno que son utilizadas como puntos de descanso y/o reunión entorno a los periodos de explotación del vertedero

Para determinar el hábitat de cada posición (con la excepción del mar, vertederos y colonia) se empleó la capa de hábitats CORINE Land Cover (España: CLC 2006; Francia: CLC 2012). Por otro lado, y con las excepciones arriba descritas, los hábitats que se han empleado en este artículo se corresponden con las siguientes categorías CORINE: prados (código 231); otro tipo de hábitats (resto de códigos).

\section{Análisis de datos}

Para el análisis de los datos no se llevó a cabo ningún filtrado. Para cada posición se determinó, primeramente, si se obtuvo de día (periodo 07:00 a 22:00) o de noche. Asimismo, se 


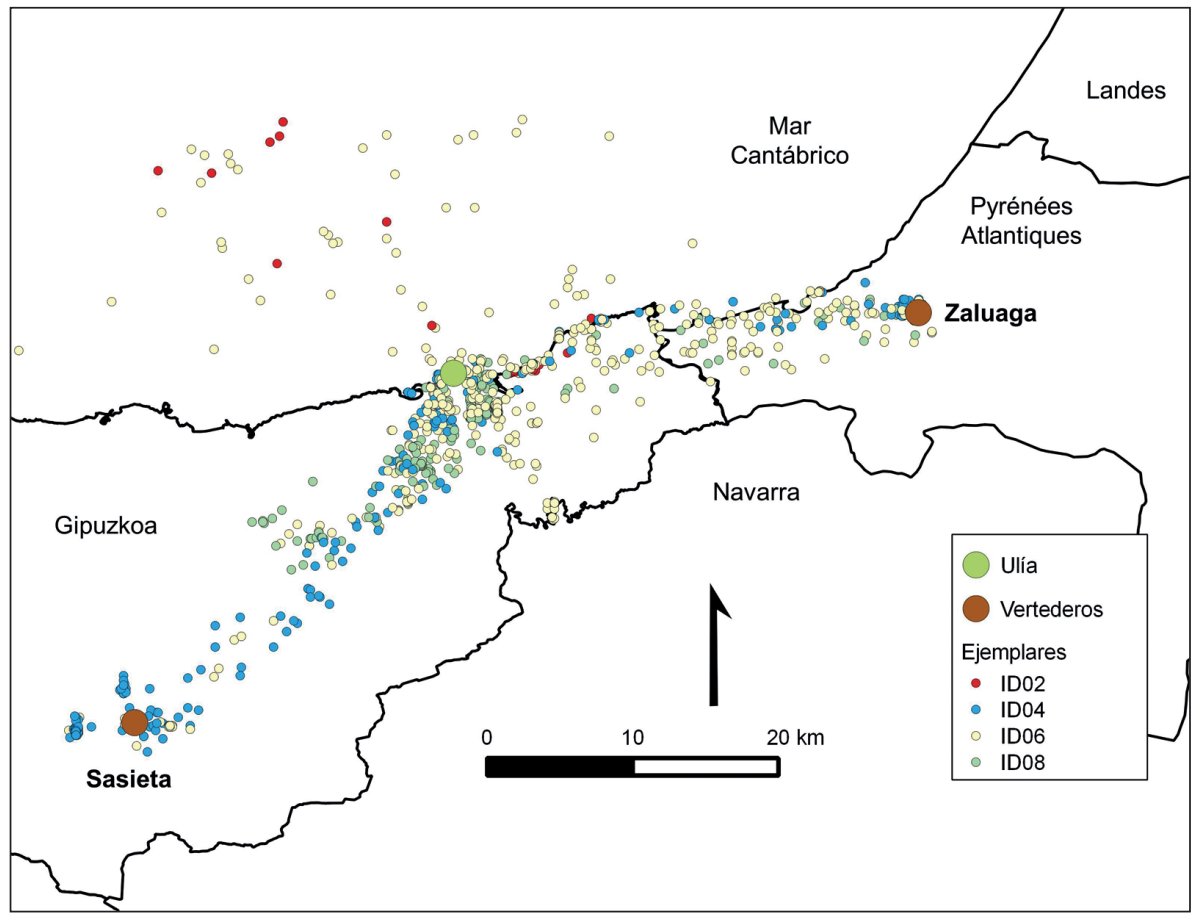

Fig. 1.- Localizaciones de cuatro ejemplares adultos de gaviota patiamarilla marcados en una de las colonias de cría en Gipuzkoa (Ulía: punto verde), durante el periodo 24.05.2016-31.07.2016. En marrón se representan los dos vertederos utilizados por las gaviotas marcadas (Sasieta y Zaluaga).

Fig. 1.- Locations of four adult yellow-legged gulls from the Ulia colony (green dot), during the period 24.05.2016-31.07.2016. The brown dots show the two landfills used by these four gulls (Sasieta and Zaluaga).

calculó la distancia a la colonia (en km). Posteriormente, determinamos el porcentaje de posiciones en cada uno de los hábitats arriba determinados. Asimismo, empleamos un ANOVA de dos factores con el fin de determinar si la distancia de los ejemplares a la colonia de cría varió entre éstos y según la hora.

La altura no se empleó para determinar si el ejemplar estaba posado o en vuelo. Esto es debido a que el rango de las alturas fue tremendamente variable y no es nada evidente determinar si el ave está posada o volando a partir de la altura a la que, además, hay que sumar el error del propio GPS. Debido al carácter preliminar y descriptivo de este artículo, se decidió no desarrollar análisis estadísticos más complejos que, tal vez, pudieran permitir esclarecer, al menos en un porcentaje de las localizaciones, si eran posiciones en el suelo o en vuelo. Estudios similares también incluyen todas las localizaciones sin distinguir si éstas eran en vuelo o posadas (Rock et al., 2016). 


\section{Resultados}

En conjunto, hasta la fecha 31.07.2016 se obtuvieron datos de 4 ejemplares (4 dispositivos): ID01, ID04, ID06 e ID08. Globalmente, se obtuvieron 2885 posiciones. El área empleada por las gaviotas (Fig. 1) abarca, en términos globales, (1) la franja de costa desde la colonia de Ulía hasta el punto de alimentación en el vertedero de Zaluaga en Senpere, (2) otra franja de territorio a lo largo de un eje que une la colonia con el vertedero de Sasieta en Beasain (con una nube de puntos densa en zonas de prados y paisaje de campiña en la zona baja de la cuenca del Urumea así como en la cuenca del río Oria, particularmente en el Asteasu), (3) un área marina de unos $1500 \mathrm{~km}^{2}$ frente a la colonia. En promedio, la superficie (polígono mínimo convexo) (Bosch et al., 2009; Rock et al., 2016) abarcada por cada ejemplar fue de $911,34 \mathrm{~km}^{2}$ (SE = 425,74 km²; rango: 307,75-2158,10 km²; Tabla 1; Fig. 2).

Globalmente, durante la noche vemos que la mayoría de las posiciones se obtuvieron en la colonia (con casi un 80\%; Tabla 2), detectándose no obstante cierto número de localizaciones en prados (10\%), así como en el mar y otro tipo de hábitats (Tabla 2). La variabilidad

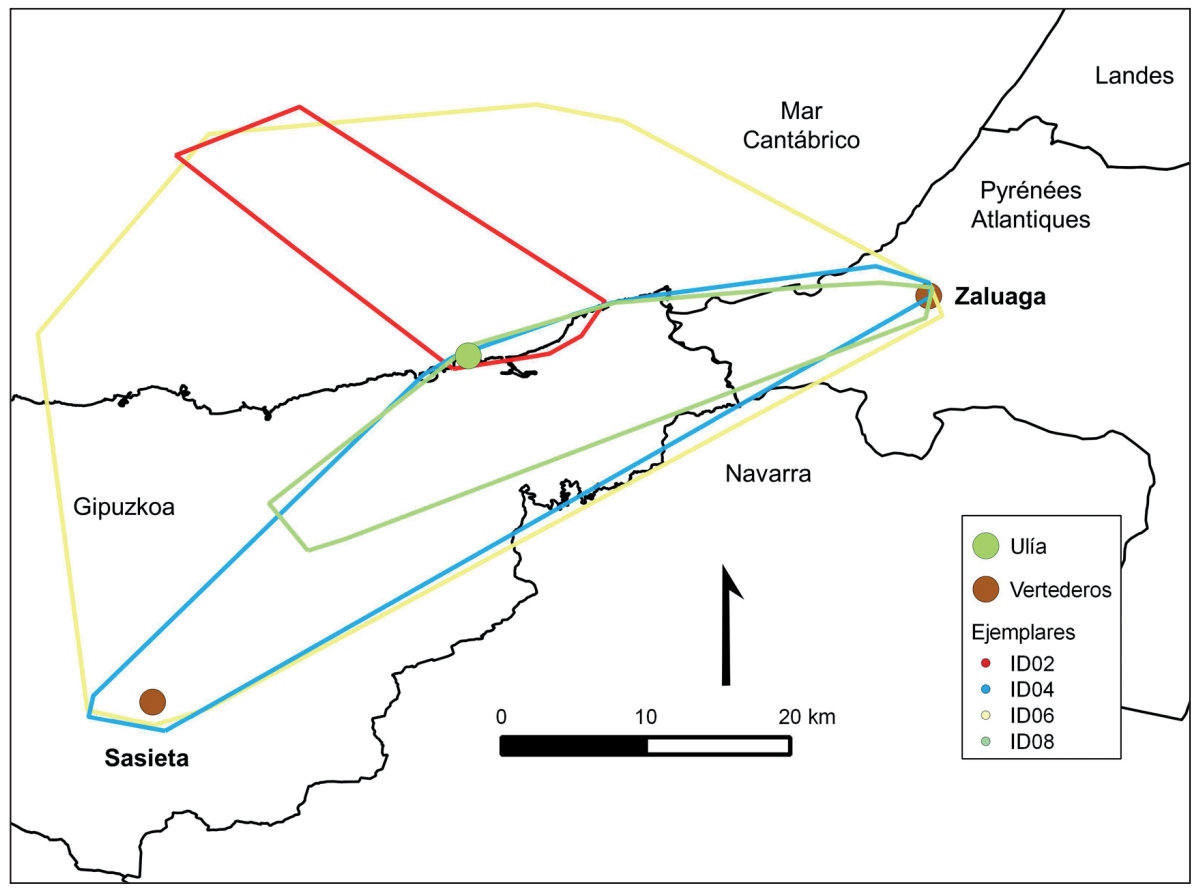

Fig. 2.-Polígono mínimo convexo de cuatro ejemplares adultos de gaviota patiamarilla marcados en una de las colonias de cría en Gipuzkoa (Ulía: punto verde), durante el periodo 24.05.2016-31.07.2016. En marrón se representan los dos vertederos utilizados por las gaviotas marcadas (Sasieta y Zaluaga).

Fig. 2.- Minimum convex polygons relative to four adult yellow-legged gulls from the Ulia colony (green point), during the period 24.05.2016-31.07.2016. Brownish points show the two open dumps used by these four gulls (Sasieta and Zaluaga). 


\begin{tabular}{|lcccc|}
\hline & ID02 & ID04 & ID06 & ID08 \\
\hline Anilla (PVC) & 6 T9:G & $564: G$ & $7 T 1: G$ & $7 T 5: G$ \\
Fecha captura & 24.05 .2016 & 02.06 .2016 & 24.05 .2016 & 02.06 .2016 \\
Fecha ultima & 30.05 .2016 & 05.07 .2016 & 29.07 .2016 & 27.06 .2016 \\
Número de días & 6 & 33 & 66 & 25 \\
Número puntos & 161 & 604 & 1450 & 670 \\
Polígono mínimo convexo $\left(\mathrm{km}^{2}\right)$ & 307,75 & 746,96 & $2.158,10$ & 432,52 \\
Sexo & $\mathrm{M}$ & $\mathrm{H}$ & $\mathrm{H}$ & $\mathrm{H}$ \\
Peso & 960 & 700 & 680 & 720 \\
Cráneo+pico & 126,46 & 115,01 & 112,62 & 115,07 \\
Cúlmen & 59,41 & 50,50 & 49,18 & 52,42 \\
Alto de pico & 22,93 & 16,13 & 16,50 & 16,47 \\
Distancia a gonys & 40,08 & 33,82 & 31,37 & 33,37 \\
Tarso & 63,21 & 57,29 & 61,49 & 60,39 \\
\hline
\end{tabular}

Tabla 1.- Características de los ejemplares capturados y marcados con GPS que proporcionaron datos durante el periodo de cría (principalmente meses de junio y julio). Toda la biometría está en mm.

Table 1.- Characteristics of the captured and GPS-tagged yellow-legged gulls which provided data during the breeding period (months of June and July). All biometrics in $\mathrm{mm}$.

individual es alta, especialmente debido a los ejemplares ID02 (para el que el 100\% de localizaciones durante este periodo se da en la colonia) e ID04 (con tan sólo un 54\% de localizaciones nocturnas en la colonia). Nótese, no obstante, que el número de las localizaciones varió mucho entre ejemplares (Tabla 1).

Durante el día, el porcentaje promedio de localizaciones en Ulía se redujo a casi un 60\%; le siguen otro tipo de hábitats (18\%) y prados y cultivos (12\%). El porcentaje global de localizaciones en vertederos es bajo (6,5\%) y observamos alta variabilidad individual (Tabla 2). El ejemplar ID02 no se localizó ni en prados ni en vertederos (si bien es el ejemplar para el que se dispone de menos datos); el resto de ejemplares sí se localizaron en prados pero utilizaron los vertederos de forma diferente. Así, mientras ID04 frecuentó ambos vertederos, el de Sasieta no fue visitado por ID08, e ID06 lo utilizó con muy baja frecuencia (Tabla 2).

En cuanto a la distancia de la colonia, observamos que las gaviotas marcadas muestran diferencias notables entre ejemplares (Fig. 3). No en vano, el modelo para determinar el patrón de distancias según horas e individuos mostró una interacción entre ambos factores (hora: $F_{14,2483}=16,179, P<0,001$; ejemplar: $F_{3,2483}=318,046, P<0,001$; horaxejemplar: $\left.F_{42,2483}=9,468, P<0,001\right)$. Globalmente, todas ellas se alejan de la colonia durante la ma- 


\begin{tabular}{|lcccccc|}
\hline Ejemplar & Ulía & Prados & Sasieta & Zaluaga & Mares & Otros \\
\hline \multicolumn{1}{l}{ Localizaciones nocturnas } & & & & & \\
\hline ID02 & 100,0 & 0,0 & 0,0 & 0,0 & 0,0 & 0,0 \\
ID04 & 54,1 & 16,2 & 0,0 & 0,0 & 16,2 & 13,5 \\
ID06 & 77,7 & 7,2 & 0,0 & 0,0 & 4,8 & 10,2 \\
ID08 & 72,6 & 17,9 & 0,0 & 0,0 & 3,6 & 6,0 \\
Total & $76,1 \pm 9,5$ & $10,3 \pm 4,2$ & - & - & $6,2 \pm 3,5$ & $7,4 \pm 2,9$ \\
& $(187)$ & $(24)$ & & & $(23)$ & $(27)$ \\
\hline Localizaciones diurnas & & & & & \\
\hline ID02 & 83,2 & 0,0 & 0,0 & 0,0 & 6,3 & 10,5 \\
ID04 & 33,8 & 17,9 & 9,8 & 7,9 & 1,9 & 28,7 \\
ID06 & 62,3 & 11,1 & 0,3 & 5,2 & 5,7 & 15,3 \\
ID08 & 57,8 & 20,6 & 0,0 & 2,7 & 1,2 & 17,6 \\
Total & $59,3 \pm 10,1$ & $12,4 \pm 4,6$ & $2,5 \pm 2,4$ & $4,0 \pm 1,7$ & $3,8 \pm 1,3$ & $18,0 \pm 3,8$ \\
& $(1098)$ & $(238)$ & $(56)$ & $(109)$ & $(99)$ & $(364)$ \\
\hline
\end{tabular}

Tabla 2.- Porcentaje de localizaciones nocturnas y diurnas según hábitat, durante el periodo de reproducción (meses de junio y julio), de 4 ejemplares adultos de gaviota patiamarilla marcados en la colonia de Ulía (Donostia). En valores totales se indica la media \pm error estándar y tamaño muestral en paréntesis (número de puntos).

Table 2.- Percentage of locations obtained both at night or during the day, according to the habitat, during the breeding period (months of June and July), of four adult gulls from the Ulia colony (Donostia). Total values show mean \pm SE and the sample size in parenthesis.

ñana, si bien la distancia que recorren varía entre individuos, así como la hora a la que se alcanzaría la máxima distancia (Fig. 3). Excepto ID04, la distancia recorrida fue sensiblemente menor de $20 \mathrm{~km}$ (<10 km en ID02). ID04, no obstante, se alejó hasta una distancia que supera los 30 km. En general se alcanza la máxima distancia de 09:00 a 14:00 (Fig. 3). La distancia máxima registrada para cada ejemplar a la colonia de cría varió entre 27,6km (ID02) a $43,0 \mathrm{~km}($ ID02).

\section{Discusión}

Este es uno de los primeros estudios en los que se analiza el uso del espacio por gaviota patiamarilla mediante dispositivos GPS (Navarro et al., 2016). Nuestros resultados son consistentes con otros estudios sobre ecología espacial en gaviotas (Bouten et al., 2013; Camphuysen et al., 2015; Tyson et al., 2015; Rock et al., 2016). Los resultados mostrados en este 

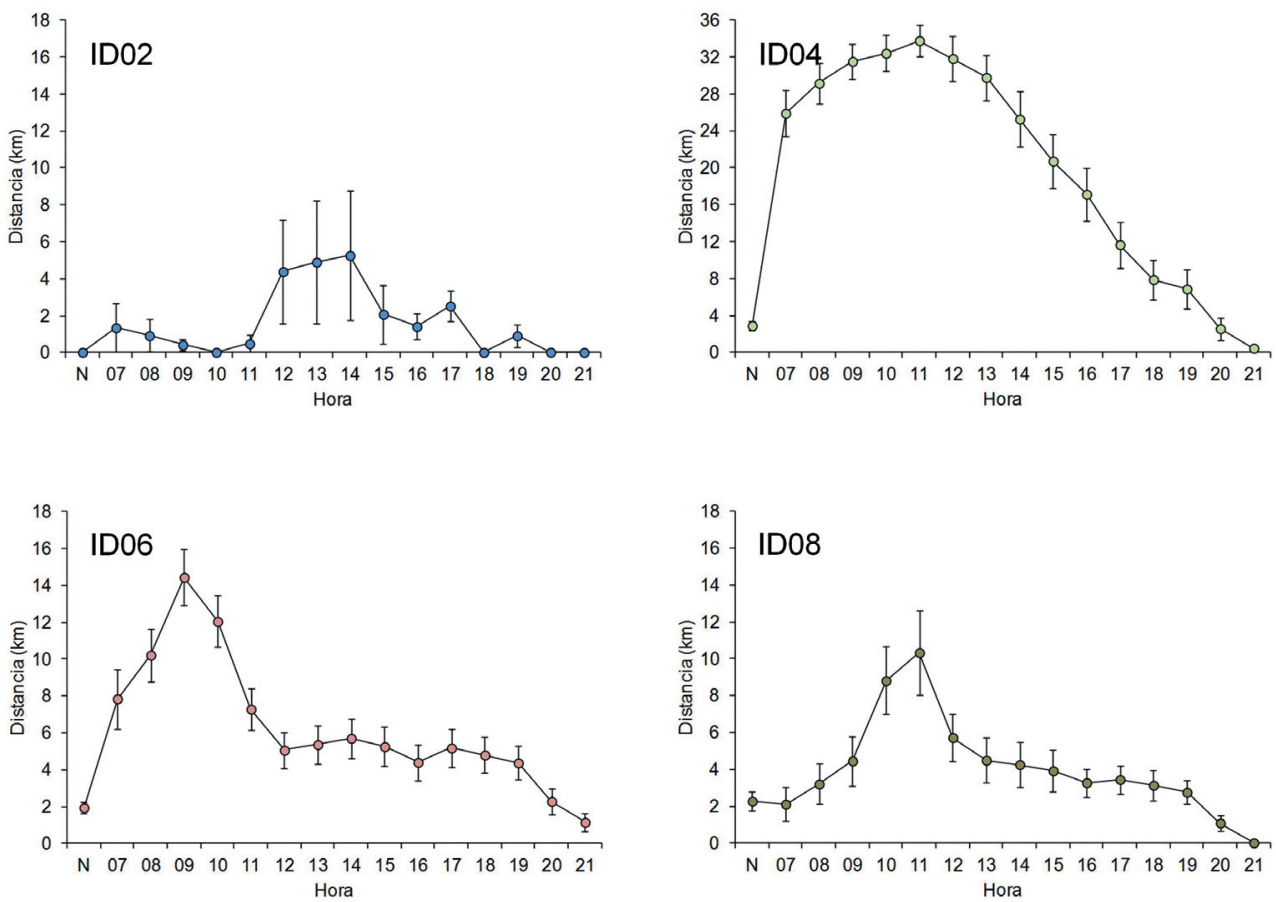

Fig.3.- Distancia (media \pm SE) a la colonia durante la noche $(N)$ y día (horas 07 a 21; 07 abarca el periodo de una hora que va de 07:00 a 08:00; 21 hace referencia al periodo de una hora que va de 21:00 a 22:00) de cada uno de los ejemplares. Nótese la diferencia de escala en el eje Y del ejemplar ID04.

Fig. 3.- Distance (mean $\pm \mathrm{SE}$ ) to the colony at night $(\mathrm{N})$ and during the day (hours 07 to $21 ; 07$ stands for the period from 07:00 to 08:00; 21 from 21:00 to 22:00) of each individual. Notice the scale difference in the $\mathrm{Y}$ axis of the individual ID04

trabajo son preliminares, ya que se dispone de datos de sólo 4 ejemplares y durante un periodo de tiempo corto. Aún así, para un periodo de monitorización inferior a 4 meses, ya disponemos de muchos más datos por ejemplar que mediante el uso de anillas de color (Arizaga et al., 2010; Juez et al., 2015).

Desafortunadamente, de 10 ejemplares marcados sólo 4 llegaron a proporcionar datos. No descartamos que, dada la altura de la vegetación en el periodo de estudio u otro tipo de factores que pudieran haber impedido la comunicación GPS-antena, pudiéramos también haber tenido datos de otros dispositivos. Adicionalmente, es muy probable que, por tratarse aún de prototipos, alguno de los dispositivos no funcionara correctamente.

Puesto que no es posible determinar con fiabilidad si las aves se encontraban posadas o volando durante las localizaciones, la interpretación del comportamiento general de las aves en cada uno de los tipos de hábitat debe hacerse con cierta cautela. En todo caso, la 
constatación en campo de aves alimentándose en los dos vertederos (Sasieta y Zaluaga) así como en prados (J. Arizaga, obs. pers.) apoyaría el hecho de que un buen porcentaje de las posiciones que proporcionan los GPS son de aves posadas, tal y como se ha asumido en estudios similares (Bouten et al., 2013; Tyson et al., 2015; Rock et al., 2016). Es más, hay ejemplares que apenas usaron los vertederos, por lo que las posiciones en los prados del entorno de la colonia podrían considerarse como puntos de alimentación y no como localizaciones situadas simplemente en la ruta hacia o desde los vertederos.

Apoyando la idea del uso de los prados, durante el periodo 2007-2009, encontramos que la dieta de las gaviotas de Gipuzkoa se componía de, aproximadamente, un 20\% de invertebrados de origen terrestre. Posteriormente, tras el cierre de cierto número de vertederos en la zona o el uso de cetrería para dificultar el acceso a gaviotas en otros (Arizaga et al., 2013a), cabe pensar que el uso de recursos tróficos alternativos, como son los invertebrados de origen terrestre (principalmente, lombrices), haya aumentado. Resultados preliminares (no publicados) basados en un estudio sobre la dieta de la población apoyan este planteamiento. En concordancia, el gran número de localizaciones en prados y cultivos apoyaría un incremento de la importancia de lombrices u otro tipo de invertebrados de origen terrestre en la alimentación de la especie durante, como mínimo, el periodo de cría y para la colonia de estudio (Ulía).

El porcentaje de localizaciones en vertederos sumó, en promedio, un 6,5\%. Esto indica que aún existe un uso de los mismos por las gaviotas de Ulía, lo que indica que la cetrería no impide el acceso a los vertederos de Sasieta y Zaluaga con una eficacia del 100\%. En todo caso, el porcentaje de localizaciones en vertederos es, comparativamente, bajo, lo cual apoyaría un uso más marginal de esta fuente de alimento en comparación con los invertebrados de origen terrestre. Será esta una cuestión que deberá corroborarse mediante el análisis de la alimentación. Entre Sasieta y Zaluaga, parece que las gaviotas usan algo más este último, lo que en cierto modo podría ser debido a que en los últimos tiempos se ha dejado de utilizar cetrería u otro tipo de medios disuasivos en este vertedero (A. Herrero, obs. pers.).

Finalmente, apenas un 4\% de las localizaciones se obtuvieron en el mar, lo cual indica que las gaviotas de la colonia de estudio, probablemente, se alimentan poco de recursos de origen marino durante el periodo de cría, al menos si hablamos de recursos que son capturados directamente en el mar, sea el caso de descartes pesqueros o no. Además, hay que considerar que un porcentaje de las localizaciones que se obtienen en el mar no serían necesariamente de puntos de alimentación, sino de descanso (Shamoun-Baranes et al., 2011). La atribución de localizaciones a puntos de descanso, en principio, no se debería aplicar al caso de los prados y cultivos, ya que las gaviotas, generalmente, no utilizan este tipo de hábitats para descansar, según hemos observado (J. Arizaga, obs. pers.)

Por otro lado, observamos una variabilidad individual muy alta en lo que al uso de diferentes hábitats se refiere, lo cual coincide con los resultados hallados en otras especies de gaviotas (Rock et al., 2016). Esto podría ser debido a varias causas. Para determinar cuál de ellas explicaría mejor nuestros datos será imprescindible aumentar el tamaño de muestra. Podemos avanzar algunas de ellas vinculadas a variación en la ecología trófica de los individuos: 
(1) la existencia de cierta especialización en la dieta a nivel individual (Oro et al., 2005; Tyson et al., 2015); (2) la variabilidad temporal en el uso de recursos y hábitats tróficos durante el periodo de cría (influyen aquí la disponibilidad de alimento a lo largo de este periodo, el tamaño y número de pollos, etc.) (Washburn et al., 2013); y (3) la posible diferenciación trófica entre sexos (Washburn et al., 2013).

En cuanto al patrón del uso del territorio a escala individual, en uno de los extremos hallamos al ejemplar ID02, un ave que apenas se alejó de la colonia y que ni siquiera visitó los vertederos así como prados y cultivos. No obstante, este ejemplar sólo proporcionó datos durante cinco días tras la captura y marcaje, por lo que su comportamiento hay que considerarlo con precaución. En este ejemplar hablamos de un ave en periodo de incubación, mientras que en los otros casos muchas de las localizaciones se produjeron cuando ya tenían pollos. En el otro extremo está el ejemplar ID04, que con diferencia fue el que más se movió a pesar de no ser el que más localizaciones proporcionó. Esta ave fue detectada en los dos vertederos y abarcó en total un área superior a 2000 km².

Desde el punto de vista de la gestión, todavía se confirma el uso de vertederos, especialmente en Zaluaga. Paralelamente, observamos una aparentemente muy alta explotación de prados, quizás sobreestimada por las localizaciones producidas durante el tránsito entre la colonia y los vertederos. Esta fuente de recursos podría contribuir a mantener niveles poblacionales todavía altos al menos si los comparamos con el descenso poblacional registrado en la vecina Bizkaia (Galarza, 2015). En el futuro, será interesante determinar hasta qué punto la explotación de recursos alternativos compensa el progresivo cierre de vertederos para mantener el tamaño actual de la población en Gipuzkoa.

En conclusión, el análisis preliminar de cuatro aves adultas de gaviota patiamarilla marcadas con GPS, durante el periodo de cría, aporta información básica sobre el uso del territorio. Se confirma la visita a dos de los vertederos más importantes de la región pese a las medidas tomadas por las administraciones para impedir o limitar el acceso de las gaviotas. Aparentemente, además, existe un uso de los prados de la zona. Por otro lado, parece que existe alta variabilidad individual en el uso del territorio y, posiblemente, en las estrategias de alimentación. Deberá ésta ser una cuestión abordada en próximos estudios.

\section{Agradecimientos}

Este estudio fue posible gracias a la financiación de la Diputación de Gipuzkoa y Wimbitek, que asumió una parte de los costes. La Diputación de Gipuzkoa autorizó la captura y marcaje de ejemplares. A. Galarza y otro revisor anónimo proporcionaron interesante comentarios que contribuyeron a mejorar una primera versión de este trabajo. 


\section{Bibliografía}

- Arizaga, J., Aldalur, A., Herrero, A., Cuadrado, J., Díez, E. , Crespo, A. 2013a. Foraging distances of a resident yellow-legged gull (Larus michahellis) population in relation to refuse management on a local scale. Eur. J. Wildl. Res. 60(2): 171-175.

- Arizaga, J., Aldalur, A., Herrero, A. , Galicia, D. 2008. Sex differentiation of Yellow-legged Gull (Larus michahellis /usitanius): the use of biometrics, bill morphometrics and wing tip coloration. Waterbirds 31: 211-219.

- Arizaga, J., Galarza, A., Herrero, A., Hidalgo, J. , Aldalur, A. 2009. Distribución y tamaño de la población de la Gaviota Patiamarilla Larus michahellis lusitanius en el País Vasco: tres décadas de estudio. Rev. Catalana d'Ornitologia 25: 32-42.

- Arizaga, J., Herrero, A., Galarza, A., Hidalgo, J., Aldalur, A., Cuadrado, J. F., Ocio, G. 2010. Firstyear movements of Yellow-legged Gull (Larus michahellis lusitanius) from the southeastern Bay of Biscay. Waterbirds 33: 444-450.

- Arizaga, J., Jover, L., Aldalur, A., Cuadrado, J. F., Herrero, A. , Sanpera, C. 2013b. Trophic ecology of a resident Yellow-legged Gull (Larus michahellis) population in the Bay of Biscay. Mar. Environ. Res. 87-88: 19-25.

- Barras, S. C. , Wright, S. E. 2002. Civil aircraft collisions with birds and other wildlife in Ohio, 1990-1999. Ohio J. Sci. 102: 2-7.

- Bertellotti, M., Yorio, P., Blanco, G. , Giaccardi, M. 2001. Use of tips by nesting Kelp Gulls at a growing colony in Patagonia. J. Field Ornithol. 72: 338-348.

- Bosch, R., Real, J., Tinto, A., Zozaya, E. , Castell, C. 2009. Home-ranges and patterns of spatial use in territorial Bonelli's Eagles Aquilla fasciata. Ibis 152: 105-117.

- Bouten, W., Baaij, E. W., Shamoun-Baranes, J. , Camphuysen, K. C. J. 2013. A flexible GPS tracking system for studying bird behaviour at multiple scales. J. Ornithol. 154: 571-580.

- Camphuysen, K. C. J., Shamoun-Baranes, J., van Loon, E. E. , Bouten, W. 2015. Sexually distinct foraging strategies in an omnivorous seabird. Mar. Biol. 162: 1417-1428.

- Castège, I., Milon, E., Lalanne, Y., d'Elbée, J. 2016. Colonization of the Yellow-legged gull in the southeastern Bay of Biscay and efficacy of deterring systems on landfill site. Estuar., Coast. Shelf Sci. 179: 207-214.

- Duhem, C., Bourgeois, K., Vidal, E., Legrand, J. 2002. Food resources accessibility and reproductive parameters of Yellow-legged Gull Larus michahellis colonies. Revue D Ecologie-La Terre Et La Vie 57: 343-353.

- Galarza, A. 2015. ¿Está disminuyendo la población de gaviota patiamarilla cantábrica Larus michahellis lusitanius Naumann, 1840? Censo 2013/2014 de Bizkaia (País Vasco). Munibe, Cienc. Nat. 63: 135-143.

- Geroudet, P. 1984. Origine mediterranéenne confirmée pour les Goélands leucophées du Léman. Nos oiseaux 37: 240.

- Gilbert, N. I., Correia, R. A., Silva, J. P., Pacheco, C., Catry, I., Atkinson, P. W., Gill, J. A. , Franco, A. A. M. 2016. Are white storks addicted to junk food? Impacts of landfill use on the movement 
and behaviour of resident white storks (Ciconia ciconia) from a partially migratory population. Mov Ecol 4: 1-13.

- Granadeiro, J. P., Brickle, P., Catry, P. 2014. Do individual seabirds specialize in fisheries' waste? The case of black-browed albatrosses foraging over the Patagonian Shelf. Animal Conserv. 17: 19-26.

- Harris, M. P. , Wanless, S. 1997. The effect of removing large numbers of gulls Larus spp. on an island population of oystercatchers Haematopus ostralegus: Implications for management. Biol. Conserv. 82: 167-171

- Juez, L., Aldalur, A., Herrero, A., Galarza, A. , Arizaga, J. 2015. Effect of age, colony of origin and year on survival of Yellow-Legged Gulls Larus michahellis in the Bay of Biscay. Ardeola 62: 139-150.

- Molina, B. E. 2009. Gaviota reidora, sombría y patiamarilla en España. Población en 2007-2009y método de censo. SEO/BirdLife. Madrid.

- Navarro, J., Grémillet, D., Afán, I., Ramírez, F., Bouten, W. , Forero, M. G. 2016. Feathered Detectives: Real-Time GPS Tracking of Scavenging Gulls Pinpoints Illegal Waste Dumping. PLoS ONE 11: e0159974.

- Olsen, K. M., Larson, H. 2004. Gulls of Europe, Asia and North America. Christopher Helm. London

- Oro, D., Bosch, M., Ruiz, X. 1995. Effects of a trawling moratorium on the breeding success of the Yellow-legged Gull Larus cachinnans. Ibis 137: 547-549.

- Oro, D., de Leon, A., Minguez, E., Furness, R. W. 2005. Estimating predation on breeding European storm-petrels (Hydrobates pelagicus) by yellow-legged gulls (Larus Michahellis). J. Zool. 265: 421-429.

- Raven, S. J., Coulson, J. C. 1997. The distribution and abundance of Larus gulls nesting on buildings in Britain and Ireland. Bird Study 44: 13-34.

- Rock, P., Camphuysen, C. J., Shamoun-Baranes, J., Ross-Smith, V. H., Vaughan, I. P. 2016. Results from the first GPS tracking of roof-nesting Herring Gulls Larus argentatus in the UK. Ringing \& Migration 31: 47-62.

- Shamoun-Baranes, J., Bouten, W., Camphuysen, C. J., Baaij, E. 2011. Riding the tide: intriguing observations of gulls resting at sea during breeding. Ibis 153: 411-415.

- Smith, G. C., Carlile, N. 1993. Methods for population-control within a Silver Gull colony. Wildlife Res. 20: 219-226.

- Steigerwald, E. C., Igual, J.-M., Payo-Payo, A., Tavecchia, G. 2015. Effects of decreased anthropogenic food availability on an opportunistic gull: evidence for a size-mediated response in breeding females. Ibis 157: 439-448.

- Tyson, C., Shamoun-Baranes, J., Van Loon, E. E., Camphuysen, K. , Hintzen, N. T. 2015. Individual specialization on fishery discards by lesser black-backed gulls (Larus fuscus). ICES J. Mar. Sci. 72(6): 1882-1891. doi:10.1093/icesjms/fsv021. 
- Washburn, B. E., Bernhardt, G. E., Kutschbach-Brohl, L., Chipman, R. B., Francoeur, L. C. 2013. Foraging ecology of four gull species at a coastal-urban interface. The Condor 115: 67-76.

- Yésou, P. 1991. The sympatric breeding of Larus fuscus, L. cachinnans and L. argentatus in western France. Ibis 133: 256-263. 